\title{
Répartition saisonnière du zooplancton en relation avec les caractéristiques environnementale dans le lac Kaby (Bongouanou, Côte d'Ivoire).
}

\author{
Nahon Mamadou FOFANA, Raphael N'doua ETILE, Gouli GOORE BI \\ Laboratoire d'Hydrobiologie, UFR Biosciences, Université Félix HOUPHOUËT-BOIGNY, 22 BP 582 Abidjan 22, Côte \\ d'Ivoire. \\ E-mail : Nahonfofana@gmail.com
}

Original submitted in on $16^{\text {th }}$ July 2019. Published online at www.m.elewa.org/journals/ on $31^{\text {st }}$ August 2019 https://dx.doi.org/10.4314/jab.v140i1.5

\section{RÉSUMÉ}

Objectifs : Cette étude vise à déterminer la répartition saisonnière du zooplancton en relation avec les caractéristiques environnementales dans le lac Kaby situé au Centre-Est de la Côte d'Ivoire dans la région du Moronou.

Méthode et résultats : Le zooplancton a été échantillonné mensuellement d'Avril 2017 à Mars 2018 à l'aide d'un filet à plancton de $60 \mu \mathrm{m}$ de vide de maille. Au total 31 taxons ont été observés (22 Rotifères, 3 Copépodes, 3 Cladocères et 3 autres organismes). Le groupe des Rotifères $(45,79 \%)$ a dominé l'abondance totale. En termes de densité, les Rotifères sont largement dominés par les Brachionidae $(51,60 \%)$. Globalement, la richesse taxonomique et l'abondance les plus fortes ont été enregistrées pendant la saison des pluies 26 taxons contre 22 taxons en saison sèche. Les variables Influençant fortement la diversité taxonomique et l'abondance sont la vitesse du courant, la conductivité, la transparence, la température.

Conclusion et application. Les caractéristiques physico-chimiques du lac Kaby déterminent une biodiversité zooplanctonique surtout marquée par la présence de 31 taxons principalement composés de rotifères $(45,79 \%)$, de copépodes $(40,98 \%)$, de cladocères $(4,61 \%)$ et des autres organismes $(8,59 \%)$. Par ailleurs, la distribution saisonnière du zooplancton est sous la dépendance des paramètres Physico-chimiques, en particulier la conductivité, la transparence, le $\mathrm{pH}$, la température, la teneur en nitrate révélant ainsi de fortes proportions d'espèces tolérantes à la pollution organique au cours de l'étude. Cette étude a permis de déterminer l'état de l'eau du lac Kaby à travers les organismes zooplanctoniques qui y vivent. Et confirme la possibilité d'utiliser les Brachionidae comme indicateur de pollution.

Mots clés : Zooplancton, diversité, abondance, pollution organique, répartition saisonnière 


\title{
Seasonal distribution of zooplankton in relation to environmental characteristics in Kaby Lake
} (Bongouanou, Ivory Coast)

\begin{abstract}
Objectives: This study aims to determine the seasonal distribution of zooplankton in relation to the environmental characteristics in Lake Kaby located in the Center-East of Côte d'Ivoire in the Moronou region.

Method and Results: Zooplankton was sampled monthly from April 2017 to March 2018 using a $60 \mu \mathrm{m}$ mesh plankton net. A total of 31 taxa were observed (22 Rotifers, 3 Copepods, 3 Cladocerans and 3 other organisms). Rotifers are the dominant group with (45.79\%) total abundance. In terms of density, Rotifers are largely dominated by Brachionidae (51.60\%). Overall, the highest taxonomic richness and abundance were recorded during the rainy season 26 taxa versus 22 taxa in the dry season. Variables strongly influencing taxonomic diversity and abundance are current velocity, conductivity, transparency, temperature.

Conclusion and application: The physicochemical characteristics of Lake Kaby determine a zooplanktonic biodiversity mainly marked by the presence of 31 taxa mainly composed of rotifers (45.79\%), copepods $(40.98 \%)$ and cladocerans $(4.61 \%$.) and other organizations $(8.59 \%)$. Furthermore, the seasonal distribution of zooplankton depends on the physico-chemical parameters, in particular the conductivity, the transparency, the $\mathrm{pH}$, the temperature and the nitrate content, thus revealing high proportions of species tolerant to organic pollution. This study made it possible to determine the water status of Lake Kaby through the zooplankton organisms that live there. And confirms the possibility of using Brachionidae as a pollution indicator.
\end{abstract}

Keywords: Zooplankton, diversity, abundance, organic pollution, seasonal distribution

\section{INTRODUCTION}

L'urbanisation et l'industrialisation croissantes des villes augmentent les charges des effluents domestiques et industrielles en matières organiques et en substances dissoutes. Dans la plus part des pays en voies de développement, ces effluents domestiques et industriels sont généralement déversés dans les écosystèmes aquatiques à proximité des villes sans traitement ou après un traitement sommaire. II en résulte à long terme une dégradation de la qualité de ces milieux, une diminution de la diversité, voir même une disparition des plans d'eau (Tchapgnouo, 2012). En Côte d'Ivoire, le "lac Kaby" est un réservoir artificiel situé dans la ville de Bongouanou, autrefois utilisée pour l'alimentation en eau potable la population de la ville de Bongouanou n'a pas échappé à cette situation. En effet, ces dernières décennies, la forte croissance démographique, l'extension de la ville et les déversements continus des effluents domestiques ont entrainé la dégradation de la qualité de ces eaux et son abandon en tant que source d'approvisionnement en eau potable des populations riveraines. Dans la gestion des écosystèmes aquatiques, en dehors des mesures des paramètres physico-chimiques, le zooplancton est utilisé comme un indicateur biologique de pollution, d'eutrophisation (Neto et al. 2014) et de changements environnementaux (Beaugrand et Reid, 2003 ; Beaugrand et Ibanez, 2004). Dans un contexte mondial de pressions anthropiques croissantes sur les écosystèmes aquatiques, conduisant à leur eutrophisation, il serait judicieux d'avoir des indicateurs pouvant servir de références pour les gestionnaires des écosystèmes aquatiques. La Côte d'Ivoire dispose des nombreux lacs et autres retenues d'eaux qui subissent les mêmes types de pressions dans un contexte climatique local. Etudier le peuplement zooplanctonique du lac Kaby, qui est un milieu dégradé, pourrait servir de base de données de références à la communauté scientifiques et aux gestionnaires de ces écosystèmes. La présente étude vise donc à constituer une base de données 
de référence pour la gestion et l'aménagement des écosystèmes aquatiques, à travers l'étude de la variation temporelle de la composition et la

\section{MATÉRIEL ET MÉTHODES}

Milieu d'étude: Le lac Kaby (figure 1) est une retenue d'eau crée pour l'alimentation en eau potable de la ville de Bongouanou au Centre-Est de la Côte d'Ivoire dans la région du Moronou. Cette zone d'étude se trouve en pleine agglomération de la ville de Bongouanou de ce fait le lac reçoit par ruissellement diffus les eaux pluviales, usées et des déchets provenant des ménages. Ce qui a suscité son abandon pour une autre source d'eau potable plus éloignée de la ville. Le lac kaby est peu profond avec une profondeur structure du peuplement zooplanctonique du lac Kaby.

moyenne de $1,15 \mathrm{~m}$ et une superficie de $3,5 \mathrm{ha}$. Le climat est composé de quatre saisons: la Grande saison de pluie (mars à juillet) ; la Petite saison sèche (le mois d'août seulement); la Petite saison des pluies (de septembre à octobre) et la Grande saison sèche : (de novembre à février).Cette étude vise, à faire l'inventaire du peuplement zooplanctonique de cette retenue d'eau et à caractériser son état par l'étude de la composition, l'abondance, et la structure de ces organismes.

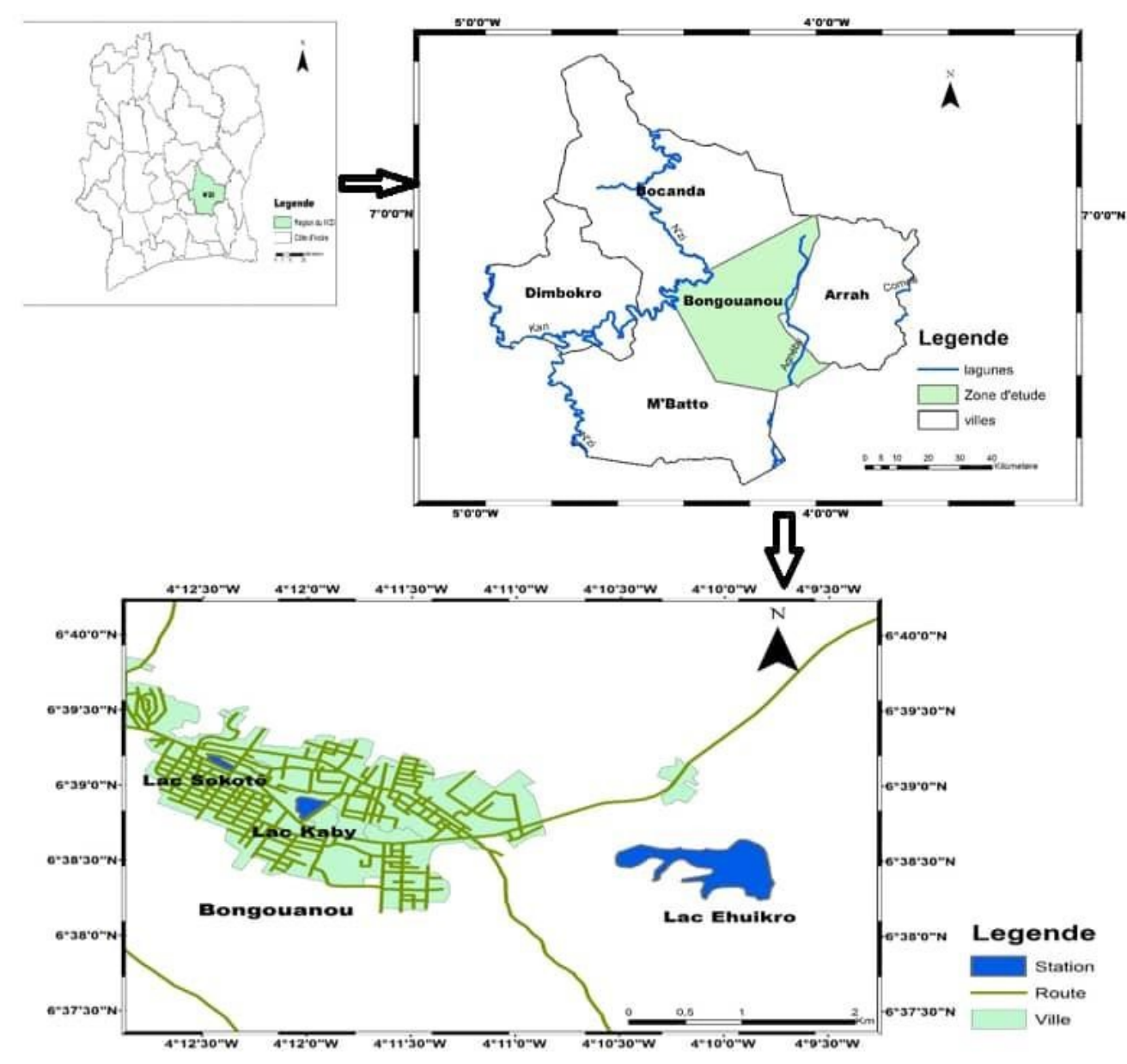

Figure 1 : Situation géographique du lac Kaby

Échantillonnage et identification des organismes zooplanctoniques : Le zooplancton a été prélevé une fois par mois, d'Avril 2017 à Mars 2018 entre 07h et 08h. Par filtration de $100 \mathrm{~L}$ d'eau (10 Seaux de capacité de $10 \mathrm{~L}$ ) à travers un filet à plancton de $60 \mu \mathrm{m}$ de vide de maille. Les échantillons récoltés ont été réduits à l'aide d'un concentreur puis récupérés dans un pilulier dans lequel ont été ajoutés 1 à $2 \mathrm{mg}$ de sucrose, 2 à 3 
gouttes de rouge neutre, 1 à 2 goutte (s) de savon liquide et du formaldéhyde (5\%). Les organismes zooplanctoniques ont été identifiés d'après les clés de POURRIOT, FRANCEZ (1986) pour les rotifères, DUSSART (1967) pour les copépodes, AMOROS (1984) pour les cladocères. Puis comptés sous une loupe binoculaire de type Vision Engineering (EW $10 \mathrm{X} / 20)$. Les résultats obtenus ont été traduits en richesse taxonomique, en occurrence (\%) $\mathrm{F}=\mathrm{Pi} / \mathrm{Pt} \mathrm{x}$ 100 ; en indice de diversité de Shannon $\mathrm{Pi}=\mathrm{ni} / \Sigma \mathrm{ni}$ \& Equitabilité $\mathrm{E}=\mathrm{H}^{\prime} / \mathrm{H}^{\prime} \max$ et exprimés en densité (ind/L).

Mesure des caractéristiques environnementales: La température de l'eau $\left({ }^{\circ} \mathrm{C}\right)$, le $\mathrm{pH}$, la conductivité $(\mathrm{GS} / \mathrm{Cm})$, le taux d'oxygène dissous (\%) ont été mesurés à l'aide d'un multi paramètre de marque Sper Scientifique. La transparence de l'eau $(\mathrm{cm})$ a été appréciée à l'aide d'un disque de Secchi. La profondeur $(\mathrm{cm})$ de l'eau a été évaluée à l'aide d'un bâton gradué. Le taux de couverture des eaux par les plantes aquatiques et le type de substrat (sable, mélange

\section{RESULTATS}

Analyse qualitative du peuplement: Au total, 31 taxons zooplanctoniques ont été recensés dans le lac Kaby. Ce peuplement comprend 22 Rotifères $(70,97 \%)$, 3 Cladocères, 3 Copépodes, et 3 autres organismes (Tableau 1). 17 familles et 21 genres ont fait l'objet de la répartition de ces taxons. Les familles les plus diversifiées sont celles des Brachionidae (8 espèces et 4 genres) suivie par les Licanidae ( 3 taxons), et les Synchaetidae, Asplanchnidae (2 taxons) les autres familles sont mono-spécifiques Parmi ces taxons observés, seuls Brachionus angularis. ( $F=75 \%)$, Brachionus calyciflorus ( $\mathrm{F}=58,33 \%$ ) Polyarthra vulgaris $(\mathrm{F}=50 \%)$, Thermocyclops dicipiens ( $\mathrm{F}=$ $83,33 \%$ ), Nauplius de copépodes ( $F=91,66 \%$ ), Larves de chironomidae $(F=75)$, Ostracode $(F=66,66)$ et les larves d'insectes $(F=91,66)$ sont constants. Tous les autres taxons sont accessoires ou accidentels. La richesse taxonomique du lac Kaby est relativement plus élevée en saison des pluies (26 taxons) qu'en saison sèche (22 taxons).

Analyse quantitative du peuplement : Le peuplement zoolactonique échantillonné dans le lac kaby se caractérise par la dominance numérique des rotifères ( $46 \%$ de l'abondance totale), suivie par les copepodes $(41 \%)$, le groupe zooplanctonique $(8 \%)$ et les cladocères $(5 \%)$. sable-gravier, gravier, boue, mélange bois mort-feuillesracines) ont été estimés par observation visuelle et exprimés en pourcentage.

Analyse des données: La richesse taxonomique, le pourcentage d'occurrence, les indices de Shannon et d'Equitabilité ont été utilisés pour déterminer la structure et la dynamique du peuplement zooplanctonique. Le pourcentage d'occurrence $(F)$ est obtenu à l'aide de la formule suivante : $F=(\mathrm{Si} / \mathrm{St}) \times$ 100 , avec $\mathrm{Si}$ : nombre d'échantillon où le taxon i a été capturé et St : nombre total des échantillons. La classification des taxons sur la base de leur pourcentage d'occurrence a été faite selon Dajoz (2000) : $F \geq 50$ : taxon constant ; $25 \% \leq F<50 \%$ taxon accessoire et $F<25$ : taxon accidentel. Les variations saisonnières des densités des organismes zooplanctoniques ont été évaluées par le test $U$ de Mann-Whitney. Les taxons zooplanctoniques et les variables environnementales ont été mis en relation avec L'analyse de redondance (RDA).

La structure de la population des copépodes est marquée par la dominance des nauplii $(59 \%)$ par rapport aux autres stades (copepodites et adultes) $(41 \%)$ représentés principalement par Thermcyclops neglectus $(25 \%)$ et Thermocyclops decipens $(16 \%)$ et les Cladocères $(4,61 \%)$. Les Rotifères sont largement dominés par Brachionus angularis $(25,62 \%)$ suivi par Brachionus calyciflorus (14,23\%).

Indice de diversité : Dans le lac kaby, la valeur la plus importante de l'indice de Shannon a été enregistré durant la saison des pluies (2,72bits/ind) et la plus faible pendant la saison sèche (1,94 bits/ind) (tableau 1). II en est de même pour l'indice d'Equitabilité $(0,83)$ pendant la saison pluvieuse contre $(0,63)$ durant la saison sèche).

Analyse mensuelle et saisonnière (Figure2). L'abondance mensuelle varie entre 9 ind/L (Octobre) et 79 ind/L (Novembre) quant à l'abondance saisonnière elle varie entre $17 \mathrm{ind} / \mathrm{L}$ (petite saison sèche) et 144 ind/L (grande saison des pluies). Par contre en moyenne les abondances de la grande saison sèche 33 ind $/ L$ et celles de la petite saison sèche ( $17 \mathrm{ind} / \mathrm{L})$ sont respectivement plus élevées que celles de la grande saison des pluies (29 ind/L) et la petite saison des pluies (10 ind/L). 


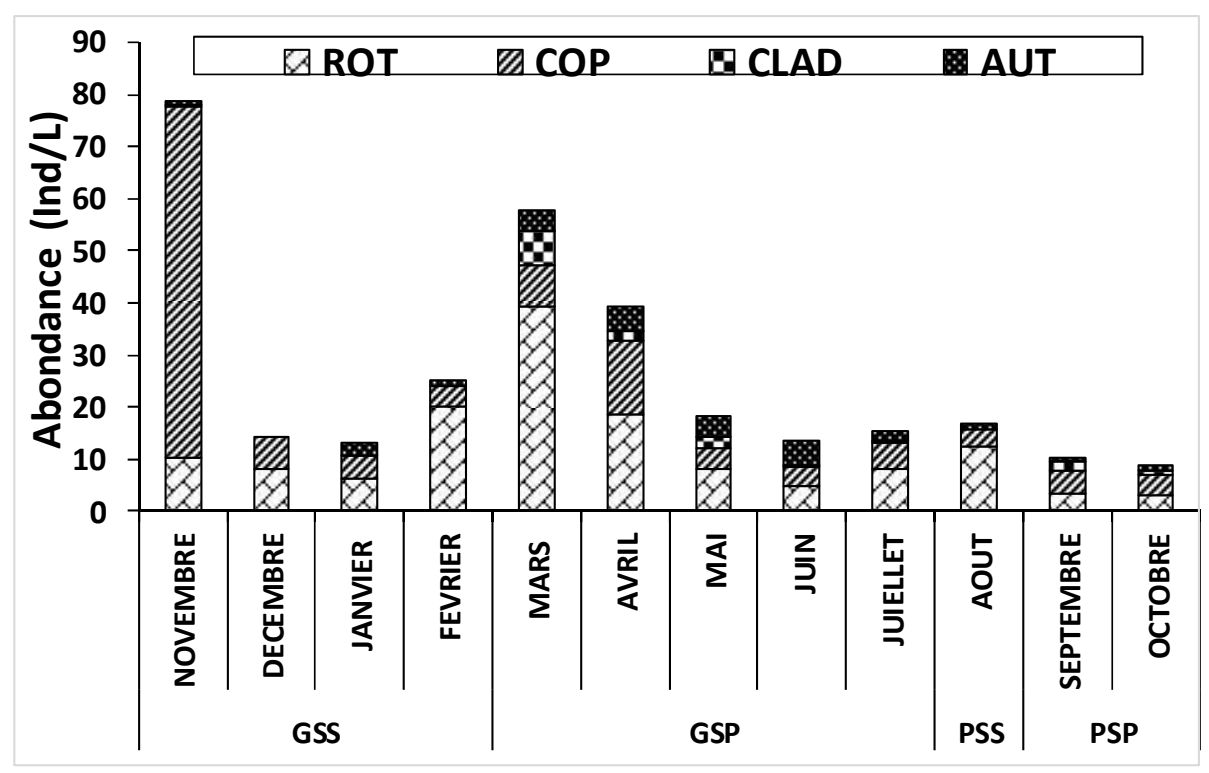

Figure 2: Variation saisonnière de l'abondance totale et de la structure du peuplement zooplanctonique échantillonné dans le lac Kaby d'Avril 2017 à Mars 2018. ROT=Rotifere; COP=Copepode; CLAD=Cladocere, AUT=Autres. GSS=Grande Saison Seche ; GSP= Grade Saisson des Pluies ; PSS =Petite Saison Sèche ; PSP=Petite Saison des Pluies.

Au niveau saisonnier, les rotifères dominent la grande saison des pluies $(55 \%)$ et la petite saison sèche $(76 \%)$. Tandis que les copépodes ont dominé le peuplement durant la grande saison sèche $(62 \%)$ et la petite saison des pluies $(40 \%)$. Si en moyenne les rotifères dominent le peuplement zooplanctonique échantillonné dans le lac kaby durant la présente étude $(46 \%)$; les copépodes sont dominants en Avril (78\%) et de septembre à novembre (37\% à $84 \%)$.

Tableau 1: Composition du peuplement zooplanctonique récolté dans le lac Kaby d'Avril 2017 à Mars 2018.

\begin{tabular}{|c|c|c|c|}
\hline GROUPES & FAMILLES & TAXA & $\%$ OCCURRENCE \\
\hline \multirow{18}{*}{ ROTIFERES } & \multirow{8}{*}{ Brachionidae } & Anuraeopsis navicula & 50 \\
\hline & & Anuraeopsis fissa & 16,66 \\
\hline & & Brachionus angularis & 75 \\
\hline & & Brachionus calyciflorus & 58,33 \\
\hline & & Brachionus plicatilis & 8,33 \\
\hline & & Brachionus falcatus & 41,66 \\
\hline & & Keratella tropica & 16,66 \\
\hline & & Plationus patulus & 16,66 \\
\hline & Filinidae & Filinia Iongiseta & 8,33 \\
\hline & \multirow{3}{*}{ Licanidae } & Lecane leontina & 25 \\
\hline & & Lecane hamata & 25 \\
\hline & & Lecane sp & 25 \\
\hline & Colurellidae & Lepadella patella & 16,66 \\
\hline & Mytilinidae & Mytilina mucronata & 8,33 \\
\hline & Gastropudae & Gastropus minor & 8,33 \\
\hline & \multirow{2}{*}{ Asplanchnidae } & Asplanchna girodi & 25 \\
\hline & & Asplanchna sp & 25 \\
\hline & Trichocercidae & Trichocerca Chattoni & 8,33 \\
\hline
\end{tabular}


Fofana et al., J. Appl. Biosci. 2019 Répartition saisonnière du zooplancton en relation avec les caractéristiques environnementale dans le lac Kaby (Bongouanou, Côte d'lvoire).

\begin{tabular}{|c|c|c|c|}
\hline & Notommatidae & Cephalodella gibba & 41,66 \\
\hline & \multirow{2}{*}{ Synchaetidae } & Polyarthra vulgaris & 50 \\
\hline & & Polyarthra remata & 16,66 \\
\hline & Hexarthridae & Hexarthra mira & 16,66 \\
\hline \multirow{3}{*}{ COPEPODES } & \multirow{2}{*}{ Cyclopidae } & Thermocyclops decipiens & 83,33 \\
\hline & & Thermocyclops neglectus & 25 \\
\hline & Indéterminée & Nauplii & 91,66 \\
\hline \multirow{3}{*}{ CLADOCERES } & Moinidae & Moina micrura & 33,33 \\
\hline & Sididae & Diaphanosoma excisum & 0,33 \\
\hline & Bosminidae & Bosmina longirostris & 8,33 \\
\hline \multirow{3}{*}{ AUTRES ORGANISMES } & \begin{tabular}{|l|} 
Chironomidae \\
\end{tabular} & Larves de chironomidae & 75 \\
\hline & Cyprididae & Ostracode & 66,66 \\
\hline & Indeterminée & autres larves d'insectes & 91,66 \\
\hline TOTAL & 17 & 31 & 31 \\
\hline \multirow{2}{*}{\multicolumn{2}{|c|}{ RICHESSES TAXONONOMIQUE }} & Saison sèche & 22 \\
\hline & & Saison des pluies & 26 \\
\hline \multirow{2}{*}{\multicolumn{2}{|c|}{ INDICES DE SHANNON }} & Saison sèche & 1,937 \\
\hline & & Saison des pluies & 2,716 \\
\hline \multirow{2}{*}{\multicolumn{2}{|c|}{ INDICES D'EQUITABILITE }} & Saison sèche & 0,6265 \\
\hline & & Saison des pluies & 0,8335 \\
\hline
\end{tabular}

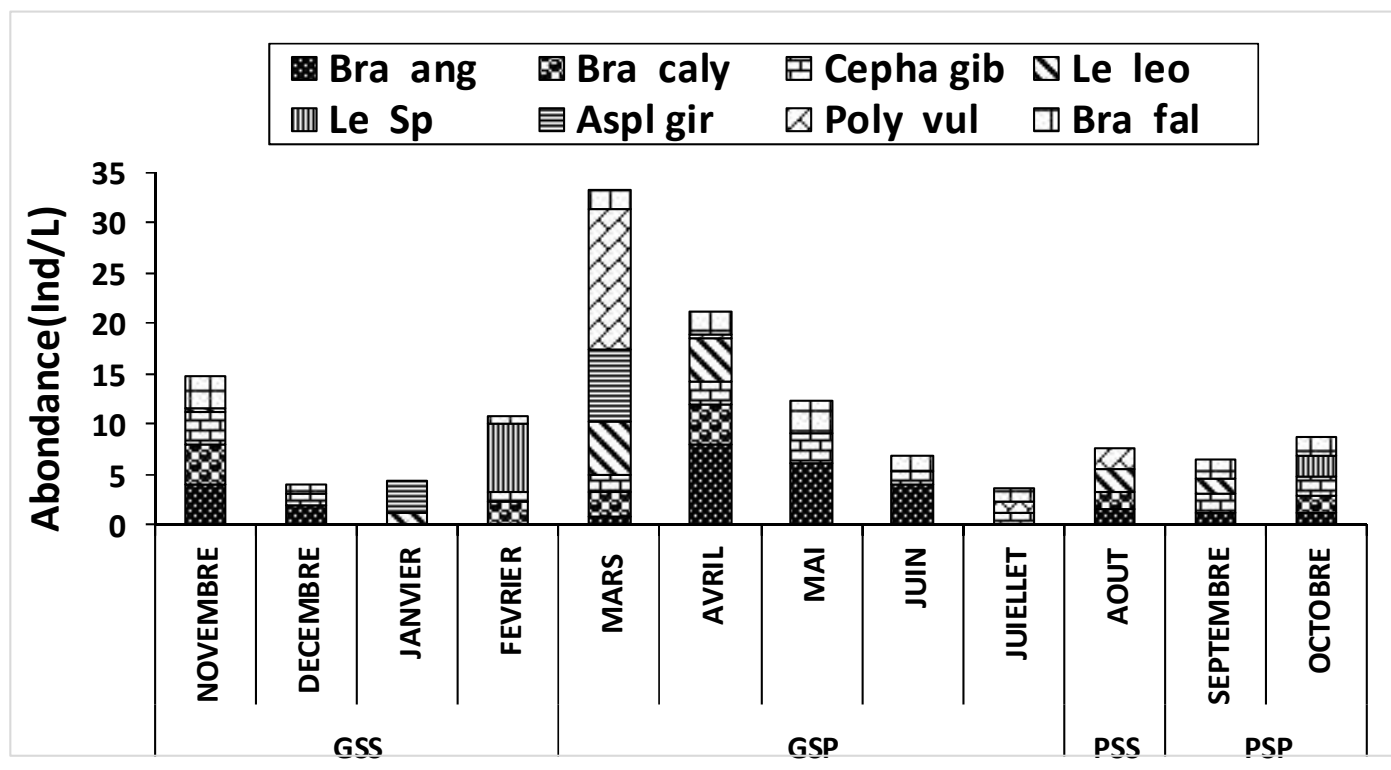

Figure 3 : Variation saisonnière de l'abondance totale et de la structure des rotifères échantillonnés dans le lac Kaby d'Avril 2017 à Mars 2018. Bra ang=Brachionus angularis; Bracaly=Brachionus calyciflorus; Bra fal= Brachionus falcatus ; Cepha gib= Cephalodella gibba ; Anu fi=Anuraeopsis fissa;Le leo=Lecane leontina ; Le sp=Lecane sp; Aspl gir=Asplanchna girodi ; Poly vul= Plyarthra vulgaris ; Hex mi= Hexarthra mira ; GSS=Grande Saison Seche ; GSP= Grade Saisson des Pluies ; PSS =Petite Saison Sèche ; PSP=Petite Saison des Pluies.

Analyse saisonnière des rotifères: (Figure 3) Les rotifères sont en moyenne dominés par 8 taxons représentants $87 \%$ de l'abondance des rotifères. Brachionus angularis (19\%); Brachionus calyciflorus
(10\%) ; Brachionus falcatus (12\%); Cephalodella gibba $(12 \%)$; Lecane leontina (10\%); Lecane sp (8\%); Asplanchna girodi (8\%); Polyarthra vulagaris (8\%). Brachionus angularis a été abondant en avril (8 ind/L) 
et mai ( 6 ind/L) Brachionus calyciflorus a été abondant en novembre ( $4 \mathrm{ind} / \mathrm{L}$ ) et avril (4 ind $/ \mathrm{L})$, Brachionus falcatus fut abondant en novembre (4ind/L) et mai (4 ind/L), Cephalodella gibba fut abondant en novembre (4 ind /L), Lecane leontina a dominé les mois de mars (5 ind/L) et avril (4 ind/L), Lecane sp a dominé les mois de décembre ( 3 ind/L) , Asplanchna girodi a été abondant en mars ( 7 ind/L) , Polyarthra vulagaris a été abondant en mas (5 ind/L).

Analyse saisonnière des Copepodes: (Figure 4) Naupliu de copépode a dominé toutes les saisons avec (76\%) des copépodes, thermocyclops decipiens a été abondant en novembre, décembre, janvier et avril (16 ind/L) et thermocyclops neglectus fut abondant en avril $(6$ ind/L).

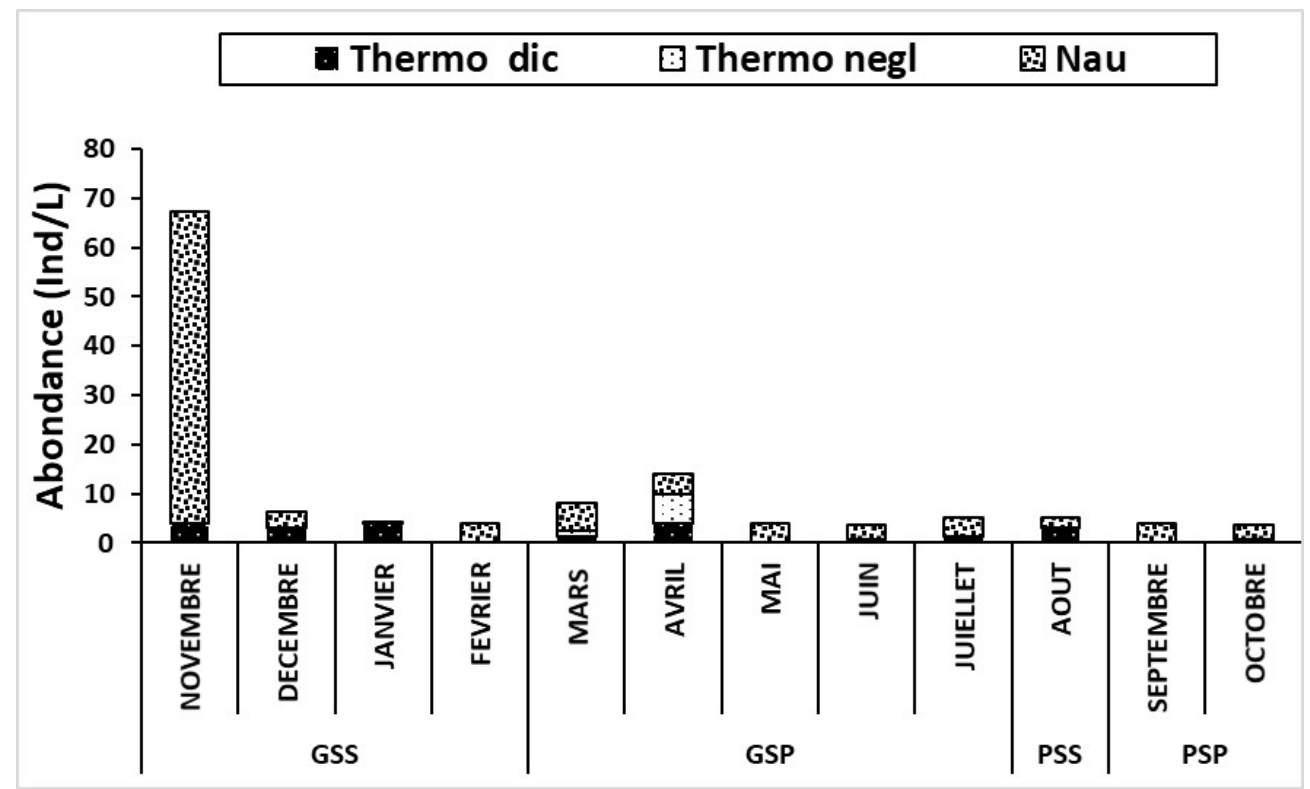

Figure 4: Variation saisonnière de l'abondance totale et de la structure des copepodes échantillonnés dans le lac Kaby d'Avril 2017 à Mars 2018. Thermo dic= Thermocyclops decipiens; Thermo negl= Thermocyclops neglectus ; Nau=Nauplii ; GSS=Grande Saison Seche ; GSP= Grade Saisson des Pluies ; PSS =Petite Saison Sèche ; PSP=Petite Saison des Pluies.

Analyse saisonnière des Cladocères:(Figure5). Diaphanosoma excisum a été plus abondant durant la grande saison pluvieuse (57\%) des cladocères, Moina micrura ( $3 \mathrm{ind} / \mathrm{L}$ ) et Bosmina longirostris (2ind/L) ont été abondant en mars.

Impact des variables abiotiques sur la répartition du peuplement zooplanctonique. (Figure 6): Les résultats de la RDA montrent que la corrélation entre les facteurs environnementaux et les taxons zooplanctoniques sont principalement expliqués par les deux premiers axes (84,2\% de la variance totale) la conductivité, la transparence, le taux de solide dissous, le taux d'oxygène, le taux de saturation d'oxygène, la profondeur, le taux de nitrate et la température sont les variables influençant fortement la distribution des organismes zooplanctoniques dans Le lac Kaby. L'ordination en RDA suivant l'axe 2 sépare nettement les saisons sèches (GSS et PSS) des saisons des pluvieuses (GSP et PSP). Le groupe des organismes: Lecane leontina, Thermocyclops decipiens, Brachionus calyciflorus, Asplanhna girodi, Anuraepsis fisa, sont corrélés a la grande saison sèche et caractérisés par le taux de solide dissous, la conductivité et la transparence. 


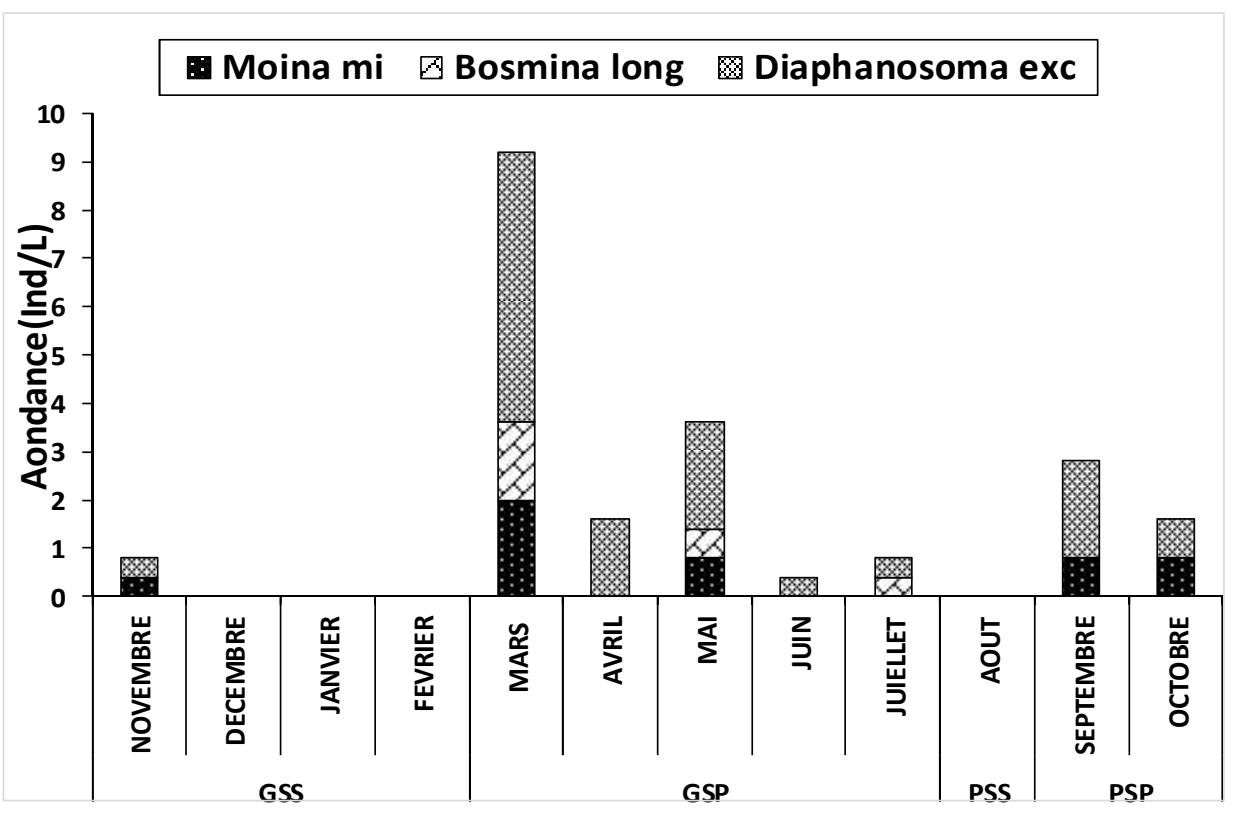

Figure 5 : Variation saisonnière de l'abondance totale et de la structure des cladocères échantillonnés dans le lac Kaby d'Avril 2017 à Mars 2018. Moina mi=Moina micrura; Bosmina long=Bosmina longirostris ; Diaphanosoma exc= Diaphanosoma excisum; GSS=Grande Saison Sèche; GSP= Grade Saison des Pluies; PSS =Petite Saison Sèche; PSP=Petite Saison des Pluies.

Le groupe des organismes: Lecane leontina, Thermocyclops decipiens, Brachionus calyciflorus, Asplanhna girodi, Anuraepsis fisa, sont corrélés a la grande saison sèche et caractérisés par le taux de solide dissous, la conductivité et la transparence. Le groupe des organismes: Polyarthra vulgaris, Hexarthra mira, Larves de chirominidae sont corrélées à la petite saison sèche et caractérisés par le taux de saturation en oxygène, l'oxygène dissous et le Potentiel d'hydrogène.
Le groupe des organismes: Bosmina longirostris, Ostracode, Naupliu et Lecane sp sont corrélés a la petite saison des pluies et caractérisés par la présence de nitrate et de la température. Le groupe des organismes: Brachionus angularis, Moina micrura et Diaphanosoma excisum sont corrélés a la grande saison des pluies et caractérisés par la profondeur et le taux de phosphore.

Tableau 2 : Valeurs moyennes et l'Ecart-type des paramètres physico-chimiques de l'eau du lac kaby mesurés d'Avril 2017 à Mars 2018

\begin{tabular}{|c|c|c|c|c|c|c|c|c|c|c|c|}
\hline & & $\begin{array}{c}\text { TDS } \\
\text { (mg/L) }\end{array}$ & $\begin{array}{c}\text { Cond } \\
\text { (Ms/cm) }\end{array}$ & $\mathrm{PH}$ & $\begin{array}{l}\text { Temp } \\
\left({ }^{\circ} \mathrm{C}\right)\end{array}$ & $\begin{array}{c}\text { Sat02 } \\
(\%)\end{array}$ & $\begin{array}{c}\mathrm{TO2} \\
(\mathrm{mg} / \mathrm{l})\end{array}$ & $\begin{array}{l}\mathrm{PO4}^{3-} \\
\text { (mg/L) }\end{array}$ & $\begin{array}{c}\mathrm{NO}^{3-} \\
\text { (mg/L) }\end{array}$ & $\begin{array}{c}\text { Transp } \\
\text { (cm) }\end{array}$ & $\begin{array}{l}\text { Prof } \\
\text { (cm) }\end{array}$ \\
\hline \multirow{2}{*}{ PSP } & MOY & 187 & 290 & 6,2 & 27,4 & 35,5 & 2,6 & 10 & 54,7 & 0,2 & 1,2 \\
\hline & Ec-Tp & 0 & 0 & 0 & 0 & 0 & 0 & 0 & 0 & 0 & 0 \\
\hline \multirow{2}{*}{ GSP } & Moy & 134,7 & 203,4 & 7,5 & 27,8 & 48,5 & 3,4 & 21,3 & 33,1 & 0,2 & 1,29 \\
\hline & Ec-Tp & 75,95 & 112,4 & 0,4 & 2,9 & 38,4 & 2,4 & 7,96 & 24,3 & 0,02 & 0,05 \\
\hline \multirow{2}{*}{ PSS } & Moy & 270,5 & 402 & 7,28 & 25,7 & 48 & 3,9 & 13,6 & 15,6 & 0,2 & 1,1 \\
\hline & Ec-Tp & 0 & 0 & 0 & 0 & 0 & 0 & 0 & 0 & 0 & 0 \\
\hline \multirow{2}{*}{ GSS } & Moy & 237,8 & 450,3 & 7,2 & 27,7 & 38,5 & 2,9 & 20,1 & 18,6 & 0,3 & 1 \\
\hline & Ec-Tp & 51,6 & 83,4 & 0,3 & 1,1 & 13,3 & 1 & 8,7 & 2,3 & 0,1 & 0,1 \\
\hline
\end{tabular}

Légende: GSP= Grande saison pluies ; GSS= Grande saison sèche ; PSS=Petite saison seche ; PSP =Petite saison pluie; Moy $=$ Moyenne; $\mathrm{Ec}-\mathrm{Tp}=$ Ecart-type $;$ Transp $=$ transparence $(\mathrm{cm}) ;$ Cond= conductivité $(\mathrm{Ms} / \mathrm{cm}) ;$ Temp $=$ température $\left({ }^{\circ} \mathrm{C}\right)$, Profd $=$ profondeur $(\mathrm{m}), \mathrm{TO} 2 \mathrm{di}=$ Oxygène dissous $(\mathrm{mg} / \mathrm{L})$, SatO2 $=$ taux de saturation en oxygène $(\%) ; \mathrm{NO}^{3-}=$ Nitrate $(\mathrm{mg} / \mathrm{L}) ; \mathrm{PO}^{3-}=$ phosphore (mg/L) ; TDS=taux de solide dissous ( $\mathrm{mg} / \mathrm{L})$. 
Tableau 3 : analyse saisonnière des paramètres physicochimiques (tableau 2)

\begin{tabular}{|l|l|}
\hline \multicolumn{2}{|c|}{ ANALYSE SAISONNIERE DES PARAMETRES PHYSICOCHIMIQUES } \\
\hline $\mathrm{TDS} \rightarrow \mathrm{SP}(135-177)<\mathrm{SS}(238-270)$ & TO2 $\rightarrow \mathrm{SP}(4-4)>\mathrm{SS}(3-4)$ \\
$\mathrm{COND} \rightarrow \mathrm{SP}(203-290)<\mathrm{SS}(402-405)$ & PO43- $\rightarrow$ SP $(10-21)>\mathrm{SS}(14-20)$ \\
$\mathrm{PH} \rightarrow \mathrm{SP}(6-7)<\mathrm{SS}(7-7)$ & NO3- $\rightarrow \mathrm{SP}(33-55)>\mathrm{SS}(16-19)$ \\
$\mathrm{Temp} \rightarrow \mathrm{SP}(27-28)<\mathrm{SS}(26-28)$ & Transp $\rightarrow \mathrm{SP}(0,2-0,2)<\mathrm{SS}(0,2-0,3)$ \\
$\mathrm{SatO} 2(\%) \rightarrow \mathrm{SP}(35-48)<\mathrm{SS}(38-48)$ & Prof $\rightarrow \mathrm{SP}(1-1,2)>\mathrm{SS}(1-1)$ \\
\hline
\end{tabular}

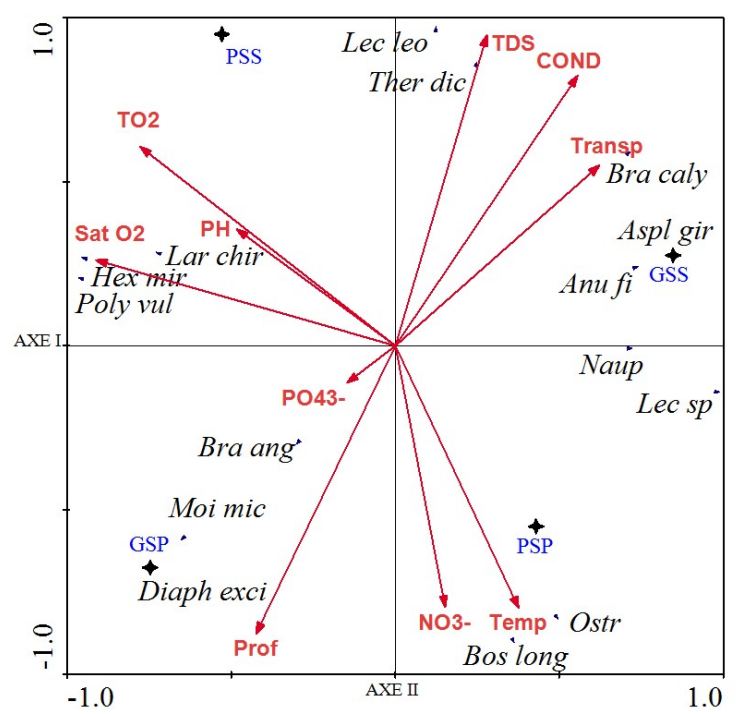

Figure 6 : Analyse de redondance (RDA) des principaux taxons du zooplancton du lac Kaby en fonction des variables environnementales. Lec leo = Lecane leontina; Ther dic= Thermocyclops decipiens, Bra caly= Brachionus calyciflorus, Aspl gir=Asplanchna girodi, Anu fisa= Anuraepsis fisa, Bos long= Bosmina longirostris, Ostr= Ostracode, Lec $\mathrm{sp}=$ Lecane sp, Poly vul $=$ Polyarthra vulgaris, Hex mir $=$ Hexarthra mira, Lar chir= Larves de chirominidae $;$ Moi mic = Moina micrura, Diaph exci= Diaphanosoma excisum ; Bra ang= Brachionus angularis.

\section{DISCUSSION}

La présente étude a permis de collecter, 31 taxons zooplanctoniques dans le lac Kaby, cette richesse pourrait s'expliquer par la petite étendue du lac avec une profondeur moyenne de 1,15 m, son emplacement en plein agglomération subissant ainsi les rejets domestiques pouvant entrainer la pollution du milieu. Cette richesse taxonomique est comparable à celle observée par Aka et al. (1998) dans les petites retenues du nord de la Côte d'lvoire (30 espèces) et Adandedjan et al. (2017) dans les données préliminaires sur la diversité du zooplancton du lac Nokoué (Sud-Bénin) (31taxons). Plusieurs raisons (caractéristiques physico-chimiques du milieu et des engins d'échantillonnage) peuvent expliquer ces observations. En effet, Ouattara et al. (2007) ont obtenu 61 taxons avec un filet à plancton de $20 \mu \mathrm{m}$ contre 30 espèces obtenues par Aka et al. (1998) avec un filet $64 \mu \mathrm{m}$ et 31 especes obtenues dans la présente étude avec un filet de $60 \mu \mathrm{m}$. les Rotifères (45,79\%) ont largement dominé le peuplement zooplanctonique. Ils constituent le groupe zooplanctonique le plus abondant en eau douce (Margalef, 1983). Les Rotifères sont capables d'ingérer des particules de petites tailles telles que les bactéries et détritus organiques souvent abondants dans les milieux eutrophes. II en résulte d'après Margalef (1983) qu'une forte représentativité des Rotifères en milieu aquatique d'eau douce peut être considérée comme un indicateur biologique d'un niveau trophique élevé. De plus, les rotifères, le principal groupe zooplanctonique $(45,79 \%)$ dans la présente étude a déjà été abondamment retrouvée dans les cours d'eau eutrophes et hypereutrophes comme le réseau hydrographique du Mfoundi (Cameroun) (Foto Menbohan, 2012). La richesse taxonomique la plus élevée a été enregistrée pendant la saison des pluies (26 taxons), ceci s'explique par 
l'arrivée des eaux pluviales avec les effluents domestiques dans la partie supérieure du lac entrainant un enrichissement du milieu en sels nutritifs favorisant la poussée algale, premier maillon alimentaire du zooplancton. La richesse taxonomique la plus faible a été enregistrée pendant la saison sèche (22 taxons) due à la période d'étiage, les organismes moins résistants ont tendance à disparaitre. Les faibles valeurs de la richesse taxonomique et de l'abondance pendant les saisons sèches ont déjà été observées par Foto Menbohan et al. (2012). La répartition dynamique des organismes zooplanctoniques dépend d'un ensemble de facteurs environnementaux dont la température de l'eau, la pénétration de la lumière, la chimie de l'eau (particulièrement le $\mathrm{pH}$, l'oxygène dissout, la salinité, les contaminants toxiques), la disponibilité de nourriture (algues, bactéries) et la prédation exercée par les poissons et les invertébrés (Onyema et Ojo, 2008). Les fluctuations de l'indice de Shannon pendant les différentes saisons (sèche= 1,937 ) et (pluvieuse $=2,716$ ) et de l'indice d'équitabilité (saison sèche $=0,6265$ ) et (saison pluvieuse $=0,8335$ ) révèlent une grande instabilité dans la structure de la communauté zooplanctonique. Ceci explique la variabilité des conditions du milieu qui y ont prévalu au cours de l'étude le cours d'eau. Cette instabilité du milieu se justifie également par la prépondérance des espèces accidentelles et/ou rares (21/30 taxons). En général, la densité et la diversité du zooplancton sont plus élevées pendant la décrue (saison sèche) que les saisons de pluies. Plusieurs conditions stables dont le mouvement des eaux, la pénétration de la lumière, la réduction des pluies observées en saison sèche pourraient favoriser le développement d'un riche spectre de zooplancton. Onyema et al. (2003), Onyema et Nwankwo (2006) au Nigéria et Okogvu (2009) au Niger ont fait des constats identiques. Toutes fois ces constats ne corroborent pas avec ceux de Saint-Jean (1983), Okogvu et Ugwumba (2006). L'abondance des cladocères: Moina micrura, Bosmina longirostis et Diaphanosoma excisum pendant les saisons pluvieuses s'explique par des températures plus basses et par des niveaux d'eau Élevés. Selon (Tchapgnouo et al. 2012) les saisons pluvieuses contribuent positivement à la croissance des populations de cladocères en apportant de nouveaux nutriments et en brassant les nutriments autochtones présents dans les différentes strates des lacs, favorisant ainsi une forte production des organismes planctoniques et le drainage de nouvelles espèces de cladocères riveraines ou recrutées dans d'autres milieux aquatiques. Par ailleurs, les rotifères $(45,79 \%)$ ont été les principaux acteurs des variations saisonnières de la diversité, avec une forte participation des Brachionidae $(51,60 \%)$. Cette dominance qualitative des rotifères est caractéristique des lacs tropicaux (Mwebaza-Ndawula et al. 2005).Généralement les espèces dominantes des genres Brachionus, Lecane et Keratella appartiennent aux familles des Brachionidae et des Lecanidae. (Ayoagui et Bonecker .2004).La richesse taxonomique (26 taxons) obtenus en saisons pluvieuses, est conforme aux résultats de Ayoagui et Bonecker (2004), mais contraire à ceux de Okogwu (2009). Selon Ayoagui et Bonecker (2004) et Okogwu (2009) le faible taux des cladocères entrainerait un manque de compétition avec les rotifères plus nombreux. En effet, selon Ayoagui et Bonecker (2004) la diminution des espèces compétitives dominantes aurait pour conséquence l'augmentation de la diversité zooplanctonique. En outre, la prédation exercée par les alevins de poissons, la mauvaise qualité de l'eau et de la compétition entre les espèces confirmeraient la baisse de la densité des espèces (Nkwoji et al. 2010). Les rotifères plus adaptés aux changements environnementales et aux pollutions organiques seraient moins affectés par certains phénomènes tel que les températures élevées et la dégradation de la qualité des eaux, contrairement aux autres organismes (Onana et al. 2014). Leur prolifération durant la saison des pluies est due à l'accumulation des nutriments dans le lac. D'où la faible richesse du milieu en organismes zooplanctoniques. La présence des nauplius de copépode très liée à au taux élevé de nitrate (moy= $44 \mathrm{mg} / \mathrm{L}$ en saison des pluies) et (moy= $17 \mathrm{mg} / \mathrm{L}$ en saison sèche) confirme que le lac subit constamment la pollution organique. Cela est similaire aux travaux de Adandedjan et al. 2017). la différence de variation de densité entre les naupliu et les copépodes pourrait s'expliquer par le cycle de développement de ces organismes. En effet, le passage des naupliu et/ou copépodites au stade adulte conduirait à une augmentation de la densité des copépodes au détriment des stades inférieurs. La prépondérance des rotifères dans le lac serait en relation avec les fortes charges en matière organique de l'eau (teneur élevée en azote ammoniacal et la conductivité). Ces matières organiques induisent une multiplication rapide des bactéries (Djuikom, 1998), qui constituent la principale source alimentaire des rotifères (Thouvenot et al. 2000). La forte présence des copépodes et des cladocères dans le lac serait liée à la 
constance saisonnière de la température (moy:27,15 ${ }^{\circ} \mathrm{C}$ ), du pH (moy : 7,04) de l'eau du milieu. Par ailleurs, la forte teneur en nitrate en saisons des pluies (moy $44 \mathrm{mg} / \mathrm{L}$ ) constatée traduit une pollution organique importante ce qui vient confirmer la présence massive des rotifères en cette saison. Même si les valeurs élevées de la conductivité (moy $426 \mathrm{Ms} / \mathrm{cm}$ ) en saisons sèches semblent contrarier la présence d'organismes zooplanctoniques dans le lac, selon certains auteurs (Saint-Jean, 1983 ; Okogvu et Ugwumba, 2006), la capacité adaptative et la tolérance de certaines espèces de copépodes (Thermocyclops, Mesocyclops et de cladocères à de grandes variations des facteurs abiotiques (conductivité, salinité et transparence) pourraient expliquer leur présence dans ce milieu. Des résultats similaires ont été obtenus par Onyema et Ojo (2008) dans les ruisseaux d'Agboyi de

\section{REMERCIEMENTS}

Les auteurs remercient Mr KOUAME Augustin, Mr KONE Yefalan pour leurs participations aux différentes campagnes d'échantillonnage et leurs conseils. Nos

\section{RÉFÉRENCES BIBLIOGRAPHIQUES}

Adandedjan D, Makponse E, Hinvi LC, Laleye P, (2017) données préliminaires sur la diversité du zooplancton du lac Nokoué (Sud-Bénin). Journal of Applied Biosciences, 115: 1147611489

Ayoagui ASM, Bonecker CC, 2004. Rotifers in different environments of the upper Parana River floodplain (Brazil): richness, abundance and the relationship to connectivity. Hydrobiologia, 522: $281-290$.

Aka N. M. 1998. Ecologie planctonique des petites retenues du Nord de la Côte-d'Ivoire. Mém. de DEA, UFR des Sciences et Gestion de l'Environnement, Université d'Abobo Adjamé, $61 \mathrm{p}$

Amoros C, 1984.Introduction pratique à la systématique des organismes des eaux continentales françaises - 5 . Crustacés Cladocères. Publication de la société linnéenne de Lyon 53-3 pp. 72-107.

BARROIN G., 1980. Eutrophisation, pollution nutritionnelle et restauration des lacs. In : Gauthier Villas éd., La pollution des eaux continentales. Incidence sur les biocénoses aquatiques, Paris, 75-96.

Beaugrand G., Brander K.M., Lindley J.A., Souissi S. \& Reid P.C., 2003. Plankton effect cod la lagune de Lagos au Nigéria, qui ont constaté une augmentation de l'abondance des rotifères et des copépodes (et copépodites) en dépit de l'augmentation rapide de la conductivité de l'eau. Par contre, selon Sacchi et Testard (1971), lorsque la conductivité de l'eau augmente, comme c'est le cas ici par l'augmentation de la salinité, la forte pression osmotique créée peut induire une migration ou une mortalité des organismes. Egalement Les pressions anthropiques exercées sur le lac auraient pour conséquences la perturbation de son intégrité biotique et abiotique. En effet, la pratique de la pêche, le transport de l'eau avec des bidons qui contenaient des produits pétroliers, le drainage des eaux dans le milieu, de même que l'occupation des espaces (habitations) constituent les principales actions anthropiques qui menacent l'intégrité de l'écosystème.

remerciements vont également aux Doctorants Apiah Saky, DIOMANDE Abou, SORO Aly pour tous les conseils lors du dépouillement et des tests statistiques.

recruitement in the North Sea. Nature, 42: 1939-1956.

Beaugrand G, lbanez F, 2004 .Monitoring marine plankton ecosystems (2): longterm changes in North Sea calanoid copepods in relation to hydro-climatic variability. Mr.Ecol.Progr.Ser, 284,35-47.

Djuikom E, 1998. Qualité bactériologique et physicochimique des cours d'eau du réseau du Mfoundi à Yaoundé. Thèse de Doctorat, Université. Yaoundé I, Cameroun, $165 \mathrm{p}$.

Dussart BH, 1982. Crustacées copepodes des eaux intérieures faunes de Madagascar 58-164p.

Dussart BH, 1967.les copepodes des eaux continentales d'Europe occidentale. 1. Calanoides et Harpacticoides 1-500 (Ed .Boubée \& Cie, Paris).

Foto Menbohan S, 2012. Recherches écologiques sur le réseau hydrographique du Mfoundi (Yaoundé) : Essai de biotypologie. Thèse de Doctorat d'État en Biologie des Organismes Animaux, Université de Yaoundé I, Cameroun, $175 p+$ annexes.

Kotov AA, Jeong HG, Lee W, 2012. Cladocera (Crustacea : Branchiopoda) of the South- East of the Korean Peninsula, with twenty new records for Korea. Zootaxa, 50-90. 
Margaleff R, 1983. Limnologia. Omega, Barcelona. $1010 \mathrm{p}$.

Mironov OG, 1972. Les effets de la pollution par les hydrocarbures sur quelques représentants du zooplancton de la mer noire, Journal of Zoology, 980-984.

Mwebaza-Ndawula L, Sekiranda SBK and Kiggundu V: 2005. Variability of zooplankton community along a section of the Upper Victoria Nile, Uganda. African Journal of Ecology, 43(3): 251257.

Nkwoji JA, Onyema IC, Igbo JK: 2010. Wet season spatial occurrence of phytoplankton and zooplankton. Science World Journal, 5(2): 714.

Onana FM, Zébazé Togouet SH, Nyamsi Tchatcho NL, Domche Teham HB and Ngassam P: 2014. Distribution spatio-temporelle du zooplancton en relation avec les facteurs abiotiques dans un hydro système urbain : le ruisseau Kondi (Douala, Cameroun). Journal. Applied. Biosciences., 82: 7326 - 7338 .

Onyema IC, Nwankwo DI, 2006. The epipelic assemblage of a polluted estuarine creek in Lagos, Nigeria. Pollution Research, 25(3): 459 -468 .

Onyema, I.C., Otudeko, O.G. and Nwankwo, D.I. 2003. The distribution and composition of plankton around a sewage disposal site at Iddo, Nigeria. Journal of Scientific Research Development, 7: 11-24

Onyema IC, Ojo AA, 2008. The zooplankton and phytoplankton biomass in a tropical creek, in relation to water quality indices. Life Science Journal, 5(4): 75-82.

Okogwu Ol: 2009. Seasonal variations of species composition and abundance of zooplankton in Ehoma Lake, a floodplain lake in Nigeria. Revista de Biologia Tropical, 58(1): 171-182.

Okogwu Ol and Ugwumba OA: 2006. The zooplankton and environmental characteristics of Ologe Lagoon, Southwest, Nigeria. Zoologist, 3: 8692

Ouattara IN, Ouattara A, Koné T, N'douba V, Gourène $G, 2007$. Distribution du zooplancton le long de deux petits bassins côtiers ouest africains (Bia et Agnébi ; Côte d'lvoire). Agronomie Africaine, 19 (2) : 197-210.

Pourriot R, 1965.Recherches sur l'écologie des Rotifères (Thèse). Vie Milieu, suppl. 21-224.
Saint-Jean L, 1983. The zooplankton. In: Lake Chad: ecology and productivity of a shallow tropical ecosystem, J.P. Carmouze, J.R. Durand Et C. Lévêque édit., Édit. W. Junk, La Haye, Monographiae Biologicae, 53: 199-232.

Thouvenot A, Debroas D, Richardot L, Jugnia B and Dévaux J: 2000. A study of changes between years in the structure of plankton community in a newly-flooded reservoir. Arvhiv für Hydrobiologia, 149: 132 - 152.

Tchapgnouo JGN, Njiné T, Zébazé Togouet SH, Segnou SCD, Tahir TSM, Tchakonté S and Pinel-Alloul B: 2012. Diversité spécifique et abondance des communautés de copépodes, cladocères et rotifères des lacs du complexe Ossa (Dizangué, Cameroun). Varia, 6: 71-93. 